\title{
Asociación del enfoque en competencia motora y habilidades motrices, con la mantención de la adherencia a la actividad física en adolescentes. Una revisión de alcance
}

\author{
Association of the focus on motor competence and motor skills, with the \\ maintenance of adherence to physical activity in adolescents. A scoping reviews
}

Cristian Oñate-Navarrete, Sofía Aranela-Castro, Christian Navarrete-Cerda y Carlos Sepúlveda-Urra

*Universidad Católica de Temuco (Chile)

Resumen. El objetivo del estudio pretende dar a conocer la asociación que existe entre el desarrollo de las habilidades motrices (HM) durante la adolescencia y la adherencia y mantención a la actividad física (AF). Metodología: Se realizó una búsqueda con el método PRISMA a partir de cinco bases de datos; Medline, Scielo, Scopus, Web of Science y Lilacs. Se consideran estudios sin restricción de diseño, desde el año 2015 al 2020, disponibles en idioma portugués, inglés, español y alemán, donde de su población de estudio incluyera adolescentes de 13 a 21 años. Se excluyeron artículos con menos de un año de intervención, fuera de los rangos etarios y que presentaran patologías. Además, no se incluyeron estudios que iniciaron su intervención en la niñez y finalizaron iniciando la adolescencia. Resultados: Se identificaron 17 estudios que abordan el enfoque de la HM para la consecución de hábitos saludables asociados a la AF y reducción del sedentarismo, los cuales demuestran que el mayor desempeño motor de los adolescentes apunta a una mayor participación en AF o deportes, pero, además, hay otros factores que parecen predecir la AF hacia futuro. Ya sea aptitud física, autoconcepto físico, la competencia motriz percibido, el consumo de medios, el peso corporal y el IMC. Conclusión: La diversificación del movimiento en las clases de educación física, influyen directamente en el mayor desarrollo de las competencias motrices en la adolescencia y la adquisición de habitos en la mantención de AF.

Palabras Claves: Adolescente; Habilidades motrices; Investigación en Educación Física; Evaluación motriz; Actividad física.

Abstract. The aim of the study is to show the association that exists between the development of motor skills (HM) during adolescence and adherence and maintenance to physical activity (PA). Methodology: A search was carried out with the PRISMA method from five databases; Medline, Scielo, Scopus, Web of Science, and Lilacs. Studies without design restriction are considered, from 2015 to 2020, available in Portuguese, English, Spanish and German, where their study population will include adolescents from 13 to 21 years of age. Articles with less than one year of intervention, out the age ranges and presenting pathologies were excluded. Furthermore, studies that began their intervention in childhood and ended in adolescence were not included. Results: 17 studies were identified that address the (HM) approach to achieve healthy habits associated with PA and reduction of sedentary lifestyle, which show that the higher motor performance of adolescents points to greater participation in PA or sports, but Furthermore, there are other factors that seem to predict PA in the future. Be it physical fitness, physical self-concept, perceived motor competence, media consumption, body weight and BMI. Conclusion: The diversification of movement in physical education classes directly influence the greater development of motor skills in adolescence and the acquisition of habits in the maintenance of PA.

Keywords: Adolescent; Motor Skills; Research in Physical Education; Motor evaluation; Physical activity.

\section{Introducción}

Existe una magnitud considerable de población adulta que requiere atención médica y cuidados permanentes anualmente, superando de esta forma, la capacidad de respuesta del sistema de salud chileno (Margozzini \& Passi, 2018). Se estiman cifras de al menos 11 millones de personas con multimorbilidad que requieren de estos cuidados de por vida (Margozzini \& Passi, 2018). Así

Fecha recepción: 22-02-21. Fecha de aceptación: 23-05-21

Cristian José Oñate Navarrete

kinecristianjose@gmail.com mismo existen cifras particularmente preocupantes en relación al aumento progresivo de la obesidad infantojuvenil, la cual en el último decenio se ha multiplicado por 10, tanto en Chile como en el resto del mundo (Margozzini \& Passi, 2018)

Se estima que en la medida que se mantengan las tendencias actuales, en 2022 aumentará la población infantil y adolescente entre los 5 y los 19 años con obesidad (OMS, 2020). Los estudios evidencian que varias morbilidades tienen su inicio durante la adolescencia (Werneck, Fernandes \& Silva 2020) en este sentido, es necesario implementar nuevas estrategias preventivas en salud, asociadas al ámbito escolar, considerando que 
el sobrepeso y la obesidad con manifestaciones secundarias de la inactividad física y que aumentan ciertas enfermedades cardiovasculares, siendo responsables de un 5\% de la mortalidad mundial (OMS, 2020). Entre los factores protectores, se encuentran la adopción y el mantenimiento de un estilo de vida activo, por ello, el desafío actual es promover efectivamente la practica de la actividad física durante las primeras dos décadas de la vida (Werneck et al., 2020).

La participación en Actividad Física (AF) está asociada con una amplia gama de beneficios para la salud, incluido el aumento de la Aptitud Cardiovascular (AC), aun así, la mayoría de los niños y adolescentes de todo el mundo, no cumple con la recomendación de al menos una hora diaria de AF para individuos de 5 a 17 años, considerando que desde los 12 años disminuye tanto la frecuencia como la participación de la AF (Hulteen et al., 2018; Huotari et al., 2018, Collins etal., 2019; Rainer \& Jarvis, 2020). Se ha sugerido que una de las explicaciones para ello, entre otros factores, se refiere a la baja adquisición temprana de habilidades motrices que pueden no desarrollarse de manera adecuada impidiendo la participación en diversas actividades y deportes (Collins et al., 2019). Por ello, la investigación se ha centrado en comprender la influencia que tienen las habilidades motrices y el aumento de la AF para promover un estilo de vida activo y saludable.

Para su descripción, la Competencia Motriz (CM) ha englobado distintas terminologías dentro de la literatura, como lo son las habilidadmes motrices (HM), habilidades fundamentales de movimiento (FMS), rendimiento motor (RM) y coordinación motora (CM) (Bardid et al., 2019). Estos términos, están definidos por la gestión y manifestación de la HM como una respuesta contextualizada, contribuyendo a que los jóvenes dispongan de un mayor repertorio de opciones motrices en la que se sientan competentes (FortVanmeerhaeghe et al., 2017).

La literatura apoya la relación entre la CM y los resultados positivos en la salud en la juventud, tales como la participación en la actividad física, la competencia física percibida, la condición física y el estado de peso con resultados que repercuten desde punto de vista, en una disminución del comportamiento sedentario, bajo riesgo cardiometabólico y un mejor estado físico, mejora en: la flexibilidad, autoestima, desarrollo cognitivo, preparación escolar y logros académicos (Bardid et al., 2019; Lopes et al., 2019, Gutiérrez et al., 2020). Por ello, se considera que el fracaso en el desarrollo de la CM probablemente resulte en opciones limitadas para una participación exitosa en la AF, perdiendo los beneficios mencionados, que son propios de la práctica de la misma (Hulteen et al., 2020; Hands et al., 2016).

Estos adolescentes con bajos niveles de CM y HM, presentan un aumento exponencial del porcentaje de grasa corporal, incremento del riesgo de peso excesivo, tendencia al estado físico disminuido y niveles más bajos de actividad y aptitud física ( Hands et al., 2016; Hands et al., 2019; Greier \& Drenowatz, 2018). Desde este punto de vista, y considerando, la asociación positiva del desarrollo de la CM, con la AF y la asociación inversa que existe hacia el comportamiento sedentario se deben propiciar las diferentes habilidades en las etapas infantiles sin desconocer a la etapa adolescente muchas veces abandonada (Vallence, 2019).

\section{Material y Método}

\section{Protocolo y registro}

Para el proceso de elaboración de la investigación, se utilizaron las directrices para revisiones sistemáticas de alcance propuesta por el reporte de recomendaciones: Extensión PRISMA para las revisiones de alcance (PRISMA-ScR) del año 2018, publicada en la Red EQUATOR. En coherencia con la guía del Joanna Briggs Institute (JBI) para las revisiones de alcance del año 2020.

\section{Criterios de elegibilidad}

Se consideraron los estudios sin restricción de diseño, que se encontraban disponibles en idioma portugués, inglés, español y alemán, traducidas por el equipo de investigación y solicitando colaboración a un revisor externo. En los que, sus años de publicación se ubicaban entre el intervalo del 2015 al 2020 y que, dentro de su población de estudio incluía adolescentes de 13 a 21 años de edad, cuya temática se relacionaba con las habilidades motrices, actividad física. Se incluyeron, además, artículos de seguimiento de resultados desde la adolescencia a la adultez. Los artículos que se excluyeron son aquellos con menos de un año de intervención, en la que su población de estudio se encontraba fuera de los rangos etarios definidos y que presentaran cualquier tipo patología. Además, no se incluyeron estudios que iniciaron su intervención en la niñez y finalizaran iniciando la adolescencia.

\section{Fuentes de información}

Se realizó una búsqueda de la literatura disponible de manera electrónica, entre abril y junio del año 2020, en relación a habilidades fundamentales del movimien- 
to, competencia motora, mantención de actividad física y beneficios cardiovasculares en adolescentes, en cinco bases de datos a saber: Medline, Scielo, Scopus, Web of Science y Lilacs, cuyo interés de resultados arrojados se sitúan en los últimos 5 años y de los cuales se registró la fecha de búsqueda más reciente, el 17 de junio del año 2020. Esta búsqueda se realizó por todos los integrantes de la investigación, utilizando un evaluador externo para resolver discrepancia en la selección de algunos artículos.

\section{Estrategia de búsqueda}

Realizada a partir del diseño PICOT, se extraen términos semejantes y descriptores en ciencias de la salud, los principales del grupo de búsqueda fueron:Adolescent [Mesh], Motor Skills[Mesh], Sedentary Behavior[Mesh] y Physical activity. Además, se utilizaron operadores booleanos para la búsqueda entre categorías y dentro de las categorías, AND y OR, respectivamente. La búsqueda para cada base de datos utilizada se adaptó a los requerimientos propios de las mismas, y el detalle de cada estrategia se presenta en la tabla 1.

Tabla 1

\begin{tabular}{|c|c|c|}
\hline \multicolumn{2}{|c|}{ Base de datosEstrategia de búsqueda } & \multirow{2}{*}{$\begin{array}{c}\begin{array}{c}\text { Año de } \\
\text { publicación }\end{array} \\
2015-2020\end{array}$} \\
\hline $\begin{array}{l}\text { MEDLINE } \\
\text { (PUBMED) }\end{array}$ & $\begin{array}{l}\text { ((((("Young Adult"[Mesh]) OR "Adolescent Health"[Mesh]) OR } \\
\text { "Adolescent"[Mesh])) AND (((("Motor Skills"[Mesh]) OR "Motor } \\
\text { skills development") OR "Motor competence") OR "Fundamental } \\
\text { movement skills")) AND (((((("Physical activity") OR "Physical } \\
\text { activities") OR "Physical exercise") OR "Physical exercises") OR } \\
\text { "Sedentary Behavior"[Mesh]) OR "Cardiorespiratory Fitness"[Mesh]) }\end{array}$ & \\
\hline$\overline{\text { SCOPUS }}$ & $\begin{array}{l}\text { (TITLE-ABS-KEY ( adolescent OR adolescence OR adolescents OR } \\
\text { teen OR teenager OR teenagers OR teens OR youth OR youths OR } \\
\text { "Adolescent Health" OR "Teen Health" OR "Young adult" OR "Young } \\
\text { adults" ) AND TITLE-ABS-KEY ( "Motor Skills" OR "Motor Skill" OR } \\
\text { "Motor competence" OR "Motor skills development" OR } \\
\text { "Fundamental movement skills" ) AND TITLE-ABS-KEY ( "Physical } \\
\text { activity" OR "Physical activities" OR "Physical exercise" OR "Physical } \\
\text { exercises" OR "Sedentary Behavior" OR "Sedentary Lifestyle" OR } \\
\text { "Physical Inactivity" OR "Cardiorespiratory Fitness" ) ) }\end{array}$ & $2015-2020$ \\
\hline $\begin{array}{l}\text { WOS } \\
\text { LILACS } \\
\text { SCIELO }\end{array}$ & $\begin{array}{l}\text { ((Adolescent OR Adolescence OR Adolescents OR "Female } \\
\text { Adolescent" OR "Female Adolescents" OR "Male Adolescent" OR } \\
\text { "Male Adolescents" OR Teen OR Teenager OR Teenagers OR Teens OR } \\
\text { Youth OR Youths OR "Adolescent Health" OR "Teen Health" OR } \\
\text { "Young adult" OR "Young adults") AND ("Motor Skills" OR "Motor } \\
\text { Skill" OR "Motor competence" OR "Motor skills development" OR } \\
\text { "Fundamental movement skills") AND ("Physical activity" OR } \\
\text { "Physical activities" OR "Physical exercise" OR "Physical exercises" } \\
\text { OR "Sedentary Behavior" OR "Sedentary Lifestyle" OR "Physical } \\
\text { Inactivity" OR "Cardiorespiratory Fitness")) }\end{array}$ & $2015-2020$ \\
\hline
\end{tabular}

\section{Proceso de extracción de datos}

Se utilizó una herramienta de abstracción de datos estandarizada diseñada para este estudio. Las categorías fueron determinadas en consenso por los autores, en coherencia con los criterios de inclusión y exclusión. El formulario comprende el ítem de elegibilidad, el cual hace referencia a los detalles de la cita del artículo, la población objetivo, que agrupa las características de los participantes, otra categoría es la intervención, que indica el tiempo de seguimiento de la publicación y por último, el foco, categoría para la descripción de la temá- tica principal, que incluye la intervención y los resultados. Este formulario sintetizó la información más relevante del estudio a fin de evaluar su idoneidad y exhaustividad, y así seleccionar los artículos atingentes para un mejor mapeo de la información, expuesto en la tabla 2 .

\begin{tabular}{|c|c|c|}
\hline \multicolumn{3}{|l|}{ Tabla 2} \\
\hline Categoría & Criterio & Descripción del criterio \\
\hline 1 población objetivo & $\begin{array}{l}\text { (1)Edad, (2) condición } \\
\text { de salud }\end{array}$ & (1)Intervalo de 13 a 21 años, (2) individuos sanos \\
\hline 2 elegibilidad & $\begin{array}{l}\text { (1)Año, (2) idioma, (3) } \\
\text { titulo }\end{array}$ & $\begin{array}{l}\text { (1)Últimos } 5 \text { años, (2) en relación a habilidades } \\
\text { motrices, (3) actividad física, y su asociación con } \\
\text { los factores de riesgo cardiovasculares. }\end{array}$ \\
\hline 3 intervención & (1)Periodo de tiempo & (1) Al menos un año. \\
\hline 4 foco & $\begin{array}{l}\text { (1)Intervención, (2) } \\
\text { resultados }\end{array}$ & $\begin{array}{l}\text { (1)Influencia de las HM en la adquisición de } \\
\text { hábitos para la realización de actividad física, (2) } \\
\text { adhesión a la AF de adolescentes por aumento del } \\
\text { desempeño de HM y en consecuencia la } \\
\text { repercusión en el sistema cardiovascular }\end{array}$ \\
\hline
\end{tabular}

\section{Síntesis de resultados}

Se recolectaron, analizaron y caracterizaron un conjunto de datos, para describir las características y el comportamiento del tema central del estudio, se resumieron los hallazgos encontrados a partir de evidencia sin restricción de diseño y a partir de ello, se realizó una descripción narrativa. En la primera fase del proceso de selección de la fuente de evidencia, se identificaron los artículos potencialmente relevantes de los cinco motores de búsqueda de bibliotecas digitales, con sus respectivas estrategias, posteriormente se eliminaron los duplicados encontrados con la aplicación web MENDELEY. Se realizó el proceso de cribado, según el análisis de las palabras contenidas en los títulos, resúmenes y palabras clave que describían el contenido de los artículos, aplicando los criterios de inclusión y exclusión para la selección, y finalmente, los estudios que pasaron el primer filtro fueron sometidos al proceso de lectura y análisis de texto completo.

\section{Calidad metodológica y verificación de calidad}

Es importante señalar que al ser una revision de alcance, esta permite tener una visión general de la evidencia existente de las habilidades motrices y su relación con la actividad física a largo plazo en adolescentes, lo cual expone los principales conceptos, teorías y brechas del conocimiento, independientemente de la calidad metodológica o el riesgo de sesgo, por lo tanto, las fuentes de evidencia incluidas en este artículo buscan entregar una visión general de este campo emergente.

\section{Riesgo de sesgo}

El método de esta revisión de alcance no fue destinado para la evaluación crítica de la evidencia encontra- 
da, por resguardo del modelo inicial de revisión narrativa, por ende, solo se agruparon hallazgos, identificaron cantidad y variedad de la información encontrada, y se entregó un sustrato de propuestas para futuras investigaciones.

\section{Resultados}

\section{Selección de fuentes de evidencia}

El resultado de la búsqueda inicial obtenida adecuada a los términos semejantes y descriptores en ciencias de la salud hasta la fecha 17 de junio del 2020, fue de 1587 artículos, de los cuales 221 corresponden a la base de datos MEDLINE, 33 a LILACS, 591 a SCOPUS, 18 artículos de SCIELO y 724 de estos a Web Of Science. Después de eliminar los duplicados, se identificaron un total de 1266, excluyendo de esta forma 329 artículos. Posteriormente basado solo en un filtro de año de publicación que se ubica desde el año 2015 al 2020, se obtuvieron 937 potenciales artículos, de los cuales según su título, resumen y palabras claves se excluyeron 841 , eliminando 89 de la base de datos MEDLINE, 12 de LILACS, 260 de SCOPUS , 16 de SCIELO y 464 de Web of Science. Los 96 artículos preseleccionados fueron sometidos a una lectura de texto completo donde se evaluó su población analizada, el periodo de tiempo de la intervención, el contenido asociado a la influencia de la $\mathrm{HM}$ en la adquisición de hábitos para la realización de AF, su adhesión a la misma y beneficios sociocognitivos. Incluyendo finalmente para esta revisión 17 artículos que se adaptaban a las categorías establecidas. De los cuales, 7 pertenecen a la base de datos MEDLINE, 3 a
SCOPUS, 7 de Web Of Science y eliminando completamente los artículos correspondientes a la base de datos SCIELO y LILACS. Proceso de selección evidenciado en el diagrama de flujo de la figura 1.

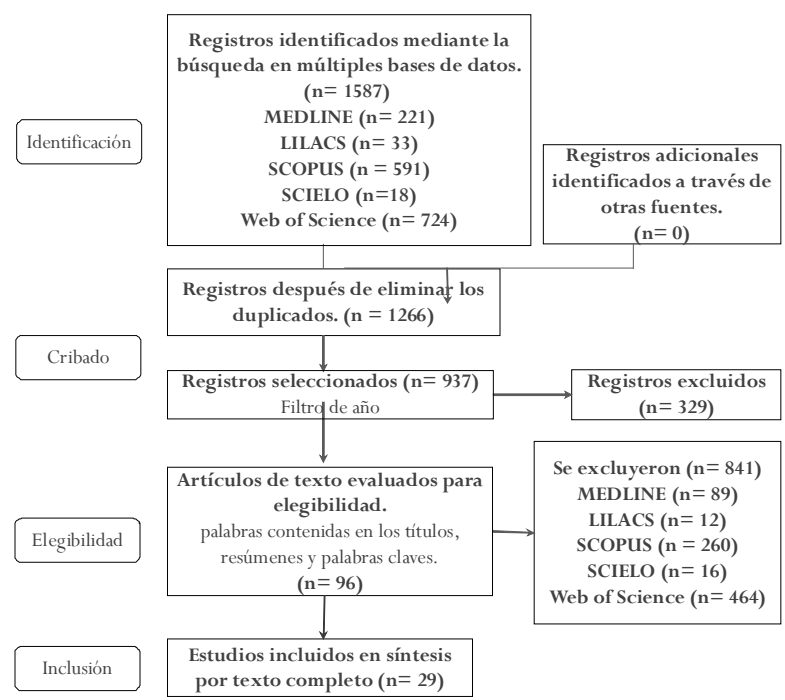

Figura 1. Resumen del proceso de selección utilizado en la revisión de recomendaciones. Directriz PRISMA-ScR año 2018.

\section{Características de las fuentes de evidencia}

Para cada fuente de evidencia, se trazaron los datos que se describen en la Tabla 3, junto al objetivo principal del estudio, la población de análisis, el tiempo de intervención, su diseño, método utilizado, medidas de resultado y los principales hallazgos en relación a las HM asociado a la AF.

Los estudios seleccionados se realizaron en 11 países diferentes, los que incluyen a dos en Irlanda, dos en Austria, dos en Portugal, dos en Finlandia, uno en Alemania, uno en Inglaterra, uno en Sudáfrica, uno en Di-

Tabla 3.

Descripción de los estudios incluidos en la revisión. Directriz PRISMA-Scr Año 2018.

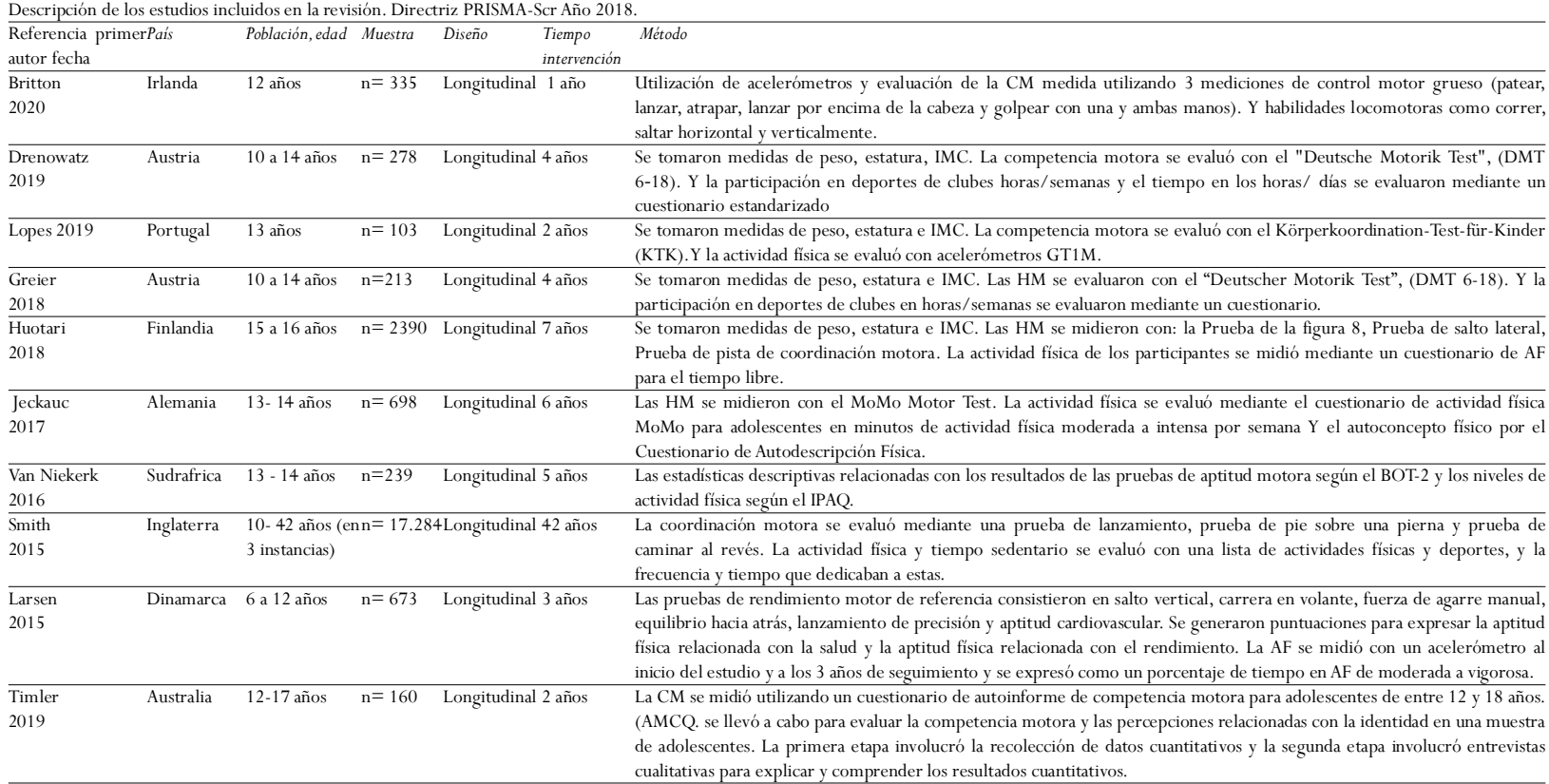


namarca, uno en Brasil, tres en Australia, uno en el Reino unido. El número de participantes en los 20 estudios incluidos osciló entre muestras de 103 y 2390 niños y jovenes de entre 12 y 22 años, incluyendo también seguimientos hasta la etapa adulta que no especifica rango etario. De todos los artículos incluidos, publicados entre los años 2015 y 2020, dos estudios pertenecen al año 2020, seis al 2019, tres al 2018, uno al 2017, tres al 2016 y dos al 2015. Por otro lado, se evaluó el diseño de los estudios seleccionados, que fueron 15 longitudinales, 1 metaanálisis y 1 revisión narrativa.

Los instrumentos para evaluar la HM utilizados en los artículos fueron variados, a continuación se describe el número de artículos y la metodología empleada: 2 artículos utilizaron elTest of Gross Motor Development (TGMD-III); 2 artículos el Deutsche MotorikTest (DMT6 18), 1 de los estudios el Körperkoordination-Test-fürKinder (KTK), 2 utilizaron la prueba de la figura 8 y además el test de salto lateral y prueba de pista de coordinación motora; el MoMo MotorTest; el BruininksOseretsky Test of Motor Proficiency Second Edition (BOT-2); el cuestionario de competencia motora adolescente (AMCQ) y la Batería de evaluación del movimiento para niños (MABC). Por otra parte, en 1 artículo se evaluó la coordinación motora mediante la prueba de lanzamiento, prueba de pie sobre una pierna y prue- ba de caminar al revés, en otro artículo se evaluó mediante la prueba de bipedestación del flamenco y la prueba de salto. Y finalmente se utilizaron las pruebas de rendimiento motor, que consistieron en salto vertical, carrera en volante, fuerza de agarre manual, equilibrio hacia atrás y lanzamiento de precisión.

La actividad física se evalúo mediante acelerómetros, el cuestionario Internacional de Actividad Física (IPAQ) y otros cuestionarios que miden la participación en clubes deportivos y AF en tiempo libre, que incluían tiempo y frecuencia destinada a realizar AF, además de listados de actividades y deportes realizados.

\section{Resultados de fuentes individuales de eviden- cia}

Para cada fuente de evidencia incluida en este estudio, se presentan los datos más relevantes en la Tabla 4, según los objetivos específicos que aborda esta revisión de alcance, a saber, sintetizar artículos que relacionan HM con la reducción de los niveles de sedentarismo, obesidad, sobrepeso y morbilidades, además de definir las variables y factores que pueden predecir significativamente la participación en la AF para la adquisición de una vida activa y finalmente evidenciar los métodos actualizados y vigentes de evaluación de la CM y de la HM aplicados en la etapa adolescente.

Tabla 4

Resultado de fuentes individuales. Directriz PRISMA-ScR año 2018.

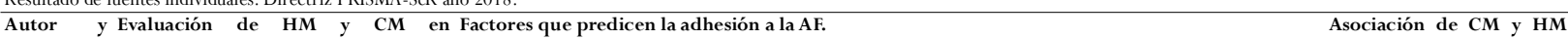

año adolescentes.

Britton TGMD-III La transición escolar está asociada con una disminución de la AF. La competencia motora el estado físico asociado a Si hay asociación

\begin{tabular}{ll}
2020 & la salud y la competencia percibida se asocia con la actividad física \\
\hline Drenowatz Deutsche Motorik Test, (DMT 6-18). & Un mayor tiempo en los medios se asoció con un mayor peso corporal y una menor participación en los deportes Si hay asociación.
\end{tabular}

2019 de club. Una mayor competencia motora al inicio del estudio se asoció con una probabilidad significativamente mayor de continuar participando en deportes de club o comenzar a participar en deportes de club, mayor de continuar participando en deportes
independientemente del peso corporal y el sexo.

\begin{tabular}{|c|c|c|}
\hline & & \\
\hline $\begin{array}{l}\text { Greier } \\
2018\end{array}$ & Deutsche Motorik Test, (DMT 6-18). & $\begin{array}{l}\text { Los resultados del presente estudio indican una asociación inversa bidireccional entre el peso corporal y las Posible asociación } \\
\text { habilidades motoras. El aumento de peso corporal se asoció con un desarrollo deficiente de las habilidades } \\
\text { motoras. Posteriormente, las habilidades motoras deficientes probablemente perjudiquen la participación en } \\
\text { deportes o actividad física general y, por lo tanto, pueden conducir a un mayor aumento de peso. }\end{array}$ \\
\hline $\begin{array}{l}\text { Lopes } \\
2019\end{array}$ & $\begin{array}{l}\text { Körperkoordination-Test-für-Kinder, } \\
\text { (KTK) }\end{array}$ & $\begin{array}{l}\text { Este estudio longitudinal encontró asociaciones positivas entre la competencia motora y la } \mathrm{AF} \text { moderada, la } \mathrm{AF} \text { de Si hay asociación. } \\
\text { moderada a vigorosa y la } \mathrm{AF} \text { total. }\end{array}$ \\
\hline $\begin{array}{l}\text { Huotari } \\
2018\end{array}$ & $\begin{array}{l}\text { Prueba de la figura } 8 \text {, Prueba de salto lateral } \\
\text { y Prueba de pista de coordinación motora. }\end{array}$ & El IMC tuvo asociaciones insignificantes con el índice de $\mathrm{AF}$ en ambos grupos de género. \\
\hline $\begin{array}{l}\text { Jakkola } \\
2016\end{array}$ & $\begin{array}{l}\text { Las HM se midieron con la prueba de } \\
\text { bipedestación del flamenco, la prueba de } \\
\text { salto y la prueba de la figura } 8 \text {. }\end{array}$ & $\begin{array}{l}\text { Las habilidades fundamentales de movimiento fueron un predictor significativo de actividad física posterior en la } S \\
\text { adolescencia. Así también la aptitud física y el IMC predijeron positivamente la actividad física de intensidad } \\
\text { moderada y vigorosa } 6 \text { años después. }\end{array}$ \\
\hline $\begin{array}{l}\text { Jeckauc } \\
2017\end{array}$ & MoMo Motor Test. & $\begin{array}{l}\text { Este estudio proporciona información sobre el papel del autoconcepto físico como predictor y mediador en la Si hay asociación, mediada } \\
\text { relación entre la actividad física y las habilidades motoras. El autoconcepto parece ser un determinante importante el autoconcepto físico. } \\
\text { de la actividad física de los adolescentes. Los efectos de la actividad física sobre las habilidades motoras son } \\
\text { parcialmente indirectos y están mediados por el autoconcepto. }\end{array}$ \\
\hline $\begin{array}{l}\text { Smith } \\
2015\end{array}$ & $\begin{array}{l}\text { Prueba de lanzamiento, prueba de pie sobre } \\
\text { una pierna y prueba de caminar al revés. }\end{array}$ & $\begin{array}{l}\text { A los } 16 \text { años no se observaron asociaciones entre la coordinación motora gruesa alta y la actividad física. La Si hay asociación, pero sólo a } \\
\text { coordinación motora gruesa solo se asoció con la participación en la actividad física a los } 42 \text { años. }\end{array}$ \\
\hline $\begin{array}{l}\text { Van Niekerk } \\
2016\end{array}$ & BOT-2 & $\begin{array}{l}\text { Los resultados muestran correlaciones entre los niveles de actividad física y los puntajes totales de competencia Si hay asociación } \\
\text { motora de este grupo de estudiantes, así como algunos ítems de prueba motora fina y los items de la prueba para } \\
\text { saltar en el lugar, botar una pelota, flexiones y abdominales. La competencia motora es un predictor de actividad } \\
\text { física en los adolescentes. Que las habilidades fundamentales de movimiento están significativamente asociadas con } \\
\text { la participación de los adolescentes en la actividad física organizada. }\end{array}$ \\
\hline $\begin{array}{l}\text { Larsen } \\
2015\end{array}$ & $\begin{array}{l}\text { Las pruebas de rendimiento motor de } \\
\text { referencia consistieron en salto vertical, } \\
\text { carrera en volante, fuerza de agarre manual, } \\
\text { equilibrio hacia atrás, lanzamiento de } \\
\text { precisión y aptitud cardiovascular }\end{array}$ & $\begin{array}{l}\text { En general, se ha informado que el componente de habilidad motora del rendimiento motor está asociado Si hay asociación } \\
\text { positivamente con la AF Lo mismo se aplica a la aptitud cardiorrespiratoria, mientras que las asociaciones con } \\
\text { otros aspectos del rendimiento motor (FMP) relacionados con la aptitud han sido pequeñas o no significativas } \\
\text { posiblemente debido a los métodos de autoinforme para medir la AF. }\end{array}$ \\
\hline $\begin{array}{l}\text { Werneck } \\
2020\end{array}$ & $\begin{array}{l}\text { Los elementos utilizados se adaptaron a los } \\
\text { requerimientos de cada artículo }\end{array}$ & $\begin{array}{l}\text { Sumando el hallazgo de Drenowatz y Greier con la evidencia disponible, parece claro que se debe priorizar la Si hay asociación } \\
\text { promoción de la actividad física desde los primeros años de vida. En la cascada de efectos, un niño activo tiende a } \\
\text { mostrar una buena competencia motora, composición corporal y ritmos biológicos normales. Por el contrario, las } \\
\text { intervenciones en la adolescencia deben dirigirse a los adolescentes de maduración temprana y aquellos con menor } \\
\text { competencia motora. }\end{array}$ \\
\hline mler & MCQ & lo \\
\hline
\end{tabular}
bienestar psicosocial y mayores problemas de conducta, hiperactividad y problemas emocionales. 


\section{Síntesis de resultados}

Los primeros resultados obtenidos de la búsqueda, se basan en entregar una definición más unificada de CM junto a los diferentes términos que engloba, como son las HM, FMS, habilidad motora, desempeño motriz, coordinación motora, y rendimiento motor. Pese a tener leves diferencias en su conceptualización, los autores sugieren la posibilidad de utilizar estos conceptos indistintamente, ya que están definidos en su mayoría como actividades motoras comunes compuestas por una serie de patrones de movimiento.

Se ha reconocido que los primeros años de la educación escolar son un momento crítico para que los niños adquieran adecuadamente las HM, obteniendo patrones de movimientos que los adhieran a la participación de AF. La mayoría de los niños son capaces de dominar desde el punto de vista del desarrollo una cantidad considerable de HM, como la carrera de velocidad, salto vertical y el lanzamiento, para las edades de 7 a 8 años y varias HM más complejas a las edades de 9 a 10 años, como saltos y conducción con los pies (O’Brien et al., 2016 ; Jaakkola et al., 2016). Por lo cual es razonable esperar que los niños (as) deban demostrar un dominio de CM antes de la transición a la escuela secundaria, vale decir, cuando inician la adolescencia alrededor de los 12 años para luego mantener los hábitos deportivos. (O’Brien et al., 2016 ; Jaakkola et al., 2016).

Como lo expuso el autor Drenowatz et al. en un estudio realizado en el año 2019, con una muestra de 278 estudiantes de quinto grado, en la cual obtuvo como resultado la probabilidad significativamente mayor de continuar participando en deportes de club o comenzar a participar en estos, independientemente del peso corporal y el sexo al aumentar la $\mathrm{CM}$ al inicio del estudio (Larsen et al., 2015; Drenowatz \& Greier, 2019).

Desde el punto de vista de la salud pública, el hecho de que la CM se asocie a la AF de moderada a vigorosa en los adolescentes es un hallazgo importante ya que, sugiere que la CM potencialmente podría usarse para aumentar los niveles de AF, lo cual es fundamental para abordar los altos niveles de sedentarismo, como lo expuso el autor Lopes et al. en su estudio publicado en 2019, que se realizó en una muestra formada por 103 adolescentes de 13 años de edad en Portugal. Este estudio longitudinal encontró asociaciones positivas entre la competencia motora y la AF moderada, la AF de moderada a vigorosa y la $\mathrm{AF}$ total, después de ajustes para las posibles covariables en los adolescentes. La proporción de $\mathrm{CM}$ en adolescentes clasificados como «regular» $\mathrm{O}$ 《 muy bueno» aumentó desde el inicio hasta el seguimiento del 62,6 al 64\% y del 2,2 al 16,9\%, respectivamente. Por el contrario, la proporción de adolescentes que cumplen con las directrices internacionales de AF de al menos $60 \mathrm{~min}$ al día de tipo moderada a vigorosa disminuyeron del 39,6 al 33\% desde el inicio hasta el seguimiento (Lopes et al., 2019)

De este mismo modo existe un estudio realizado por Greier et al. (2018) haciendo énfasis en el desarrollo de las HM en edades tempranas, postulando esto como un componente importante dentro de las medidas preventivas para abordar el sedentarismo, evitando un círculo vicioso de baja actividad física, aumento de peso corporal y HM deficientes, demostrando que un patrón elevado en las HM, contribuyen a la pérdida de peso en los niños, que inicialmente fueron clasificados con sobrepeso u obesidad.

Este estudio fue realizado en una muestra de 213 estudiantes de secundaria con rango etario de 10 a 14 años, en Austria, donde se realizó un seguimiento por 4 años. Los jóvenes fueron sometidos a la evaluación de 5 elementos de prueba para registrar la resistencia, la potencia, la velocidad, la coordinación y la agilidad. El resultado del presente estudio indica la asociación inversa bidireccional entre el peso corporal y la HM. El aumento de peso corporal se asoció con un desarrollo deficiente de la HM siendo un factor perjudicial en la participación deportiva o AF general y, por lo tanto, pueden conducir a un mayor aumento de peso (Greier \& Drenowatz, 2018).

Existe consenso entre estudios que la inactividad física es un problema que puede estar vinculado a varios factores, dentro de los cuales incluye las dimensiones psicológicas, sociales, biológicas y ambientales como lo demuestran Timler et al., (2020) y Werneck et al., (2020) en 2 estudios realizados en adolescentes, donde relacionaron las HM con factores psicológicos que pueden contribuir o perjudicar la adhesión a la AF, ya que se evidencia que las personas con menor $\mathrm{CM}$ a menudo presentan una menor autoeficacia y, por lo tanto, tienden a reducir las oportunidades de participación en AF. Además los que tienen mayor CM participan en una amplia gama de actividades que conducen a una mayor aceptación social y a un sentido positivo de sí mismo (Collins et al., 2019)

En este mismo marco se evidencian resultados que apoyan la suposición de que los efectos de la HM sobre la AF no son directos, sino que están mediados por el autoconcepto, debido a que, al parecer es un determinante importante de la AF en los adolescentes, especialmente en el período de transición entre la adoles- 
cencia y la edad adulta joven, las intervenciones destinadas a aumentar el autoconcepto positivo son prometedoras, es lo que se expone en el estudio de Jekauc et al., (2017).

En este trabajo se incluye un estudio importante, cuyo objetivo fue investigar la asociación longitudinal entre las HM en la infancia y AF, junto al comportamiento sedentario en la edad adulta, desarrollado por Smith et al. en el año 2015, en una población de 17.284 participantes nacidos en Inglaterra, Escocia y Gales, a quienes se les realizó un seguimiento hasta la adultez, incorporando datos desde los 10,16, hasta la edad de 42 años. Expuso la asociación positiva entre una mayor HM y la participación en la AF. Justificó esta asociación en la posibilidad de que los jóvenes con baja HM no encuentren agradable la participación en la AF, por ser considerado un desafío más complejo en comparación con aquellos que tienen un correcto desarrollo de las mismas. Por otro lado también destacó esta asociación de manera inversa, es decir el tiempo sedentario de manera excesiva puede inhibir el desarrollo de la HM, perjudicando de igual manera la adhesión a la AF en las siguientes etapas del ciclo vital.

\section{Conclusión}

En esta revisión de alcance se encontró que sí existe asociación entre la HM de los adolescentes y la adherencia a la $\mathrm{AF}$ en el futuro, a pesar de que, los determinantes y factores de la participación de AF entre los adolescentes son variados y no se encuentran totalmente identificados y establecidos (Hardy, 2018).

Con base a lo expuesto existe una vasta evidencia de cuestionarios realizados a la población infantil y en menor cantidad de cuestionarios y escalas de valoración que permiten precisamente cuantificar la HM de forma específica en adolescentes. La elección del método de evaluación a utilizar depende de una serie de aspectos que incluyen el propósito de la evaluación, las características de la población, los aspectos administrativos y la calidad de la medición. Dentro de estos métodos evaluativos destacan, Test of Gross Motor Development (TGMD-III), el Deutsche Motorik Test (DMT 6 18), el Körperkoordination-Test-für-Kinder (KTK), el MoMo Motor Test, la batería de evaluación del movimiento para niños (MABC), Bruininks-Oseretsky Test of Motor Proficiency Second Edition (BOT-2), MAND, ACM y el cuestionario de CM del Adolescente (AMCQ) (Britton et al, 2020; O’Brien et al., 2016; Drenowatz \& Greier, 2019; Greier \& Drenowatz, 2018; Lopes et al., 2019;
Hands et al., 2016)

Además se ha proporcionado evidencia que el desarrollo de las HM tiene una influencia positiva en los niveles de AF en años posteriores, además de la mantención de un peso corporal saludable en la edad adulta (Greier \& Drenowatz, 2018; Utesch, 2019; Huotari, 2018; Martin, 2021). De modo que, las acciones de infancia y juventud realizadas durante este periodo van a repercutir en la mantención de estos hábitos en las siguientes etapas después de la adolescencia, como lo es la adquisición de un estilo de vida saludable por la realización placentera de AF (Martin, 2021; Marín \& López, 2020).

Considerando esto, el enfoque en las HM podría usarse para aumentar los niveles de AF en niños y adolescentes, al crear hábitos saludables para la adherencia y práctica de la actividad física y deporte en la etapa adulta. Además, los adolescentes con una menor competencia motora deberían tener la opción de un reforzamiento o entrenamiento individualizado para experimentar una amplia variedad de movimientos los que mejorarían su CM y confianza en las clases de educación física escolar, sin embargo los instrumentos actuales utilizados en la enseñanza escolar chilena solo evaluan Aptitud física y componentes aislados de ciertas habilidades motrices ( Oñate et al., 2020).

\section{Discusión}

En esta revisión de alcance, se identificaron 17 estudios que abordan el enfoque en las HM para la consecución de hábitos saludables asociados a la AF y reducción del sedentarismo. Los resultados muestran que el mayor desempeño motor de los adolescentes apunta a una mayor participación en actividades físicas o deportes, pero, además, hay otros factores que parecen predecir la actividad física hacia futuro, entre los cuales se encuentran la aptitud física, el autoconcepto físico, la competencia motriz percibida, el consumo de medios, el peso corporal y el IMC.

Estos hallazgos concuerdan con los de Lima et al., (2017) donde los resultados de su estudio longitudinal de 6 años indican que la competencia motora y la actividad física tienen una relación longitudinal recíproca. Cabe destacar que la competencia motora fue evaluada en la infancia y no en la adolescencia, ya que el estudio consideró a niños de 6 años, que luego revaluados a los 13 años. También Lubans et al., (2010) identificaron en su revisión sistemática una fuerte evidencia de una asociación positiva entre la competencia motora y la activi- 
dad física en niños y adolescentes en sus estudios transversales, pero no así en su estudio longitudinal en donde no se encontró asociación directa. Estos resultados dan indicios de que la competencia motora puede ser un factor que predice la actividad física a futuro, pero faltan estudios de seguimiento que consideren como población a los adolescentes y evalúen sus efectos en la etapa adulta.

Al igual que en el presente estudio, los resultados de Hulteen et al., (2018) en su revisión sistemática indican que el peso corporal es un factor importante que influye en el desempeño motriz de los adolescentes, ya que, los participantes con peso normal tenían un dominio significativamente más alto en el salto horizontal, balanceo y equilibrio en comparación con los participantes con sobrepeso y obesidad (Rainer \& Jarvis, 2020). Esto apoya la idea que el peso y la HM tienen una relación recíproca.

\section{Futuras líneas de investigación}

Las futuras líneas de investigación deben ir orientadas a realizar estudios con mayor tiempo de seguimiento, considerando mediciones en la infancia, adolescencia y adultez, para poder concretar resultados verídicos y no estimativos, realizando las evaluaciones con test estandarizados para la etapa adolescente y considerando los aspectos psicológicos y sociales que inciden sobre los adolescentes, debido a que los estudios analizados hasta la fecha no incluyen mayor información de aquello.

\section{Referencias}

Bardid, F., Vannozzi, G., Logan, S., Hardy, L., \& Barnett, L. (2019).A hitchhiker's guide to assessing young people's motor competence: Deciding what method to use. Journal of science and medicine in sport, 22(3), 311-318.

Britton, U., Issartel, J., Symonds, J., \& Belton, S. (2020). What keeps them physically active? predicting physical activity, motor competence, health-related fitness, and perceived competence in irish adolescents after the transition from primary to second-level school. International journal of environmental research and public health, 17(8), 2874.

Castelli, D. \& Valley, J. (2007). Chapter 3:The relationship of physical fitness and motor competence to physical activity. Journal of Teaching in Physical Education, 26(4), 358374.

Collins, H., Booth, J., Duncan,A., \& Fawkner, S. (2019).The effect of resistance training interventions on fundamental movement skills in youth: a meta-analysis. Sports medicine-open, 5(1), 1-16.
Drenowatz, C., \& Greier, K. (2019). Cross sectional and longitudinal association of sports participation, media consumption and motor competence in youth. Scandinavian journal of medicine \& science in sports, 29(6), 854-861.

Fort-Vanmeerhaeghe, A., Román-Viñas, B., \& Font-Lladó, R. (2017). ¿ Por qué es importante desarrollar la competencia motriz en la infancia y la adolescencia? Base para un estilo de vida saludable. Apunts. Medicina de l'Esport, 52(195), 103-11

Greier,K., \& Drenowatz, C. (2018). Bidirectional association between weight status and motor skills in adolescents. Wiener klinische wochenschrift, 130(9), 314320.

Gutiérrez, S., de Cos, I., Galarraga, S., \& de Cos, G. (2020). Evaluación de la precisión de percepción de competencia motriz en adolescentes. Publicaciones: Facultad de Educación y Humanidades del Campus de Melilla, 50(1), 341-355.

Hands, B., Chivers, P., Grace,T., \& McIntyre, F. (2019).Time for change: Fitness and strength can be improved and sustained in adolescents with low motor competence. Research in developmental disabilities, 84, 131 138.

Hands, B., Parker, H., Rose, E., \& Larkin, D. (2016). Gender and motor competence affects perceived likelihood and importance of physical activity outcomes among 14 year olds. Child: care, health and development, 42(2), 246-252.

Hardy, L., Ding, D., Peralta, L., Mihrshahi, S., \& Merom, D. (2018). Association between sitting, screen time, fitness domains, and fundamental motor skills in children aged 5-16 years: cross-sectional population study. Journal of Physical Activity and Health, 15(12), 933-940

Hulteen, R. M., Morgan, P. J., Barnett, L. M., Stodden, D. F., \& Lubans, D. R. (2018). Development of foundational movement skills: A conceptual model for physical activity across the lifespan. Sports Medicine, 48(7), 1533-1540.

Hulteen, R., Barnett, L., Morgan, P., Robinson, L., Barton, C., Wrotniak, B., \& Lubans, D. (2018). Development, content validity and test-retest reliability of the Lifelong Physical Activity Skills Battery in adolescents. Journal of sports sciences, 36(20), 2358-2367.

Hulteen, R., True, L., \& Pfeiffer, K. (2020). Differences in associations of product-and process-oriented motor competence assessments with physical activity in children. Journal of sports sciences, 38(4), 375-382.

Huotari, P., Heikinaro Johansson, P., Watt, A., \& Jaakkola,T. (2018). Fundamental movement skills in adolescents: Secular trends from 2003 to 2010 and associations with physical activity and BMI. Scandinavian journal of medicine \&science in sports, 28(3), 1121-1129.

Jakkola,T.,Yli Piipari, S., Huotari, P.,Watt,A., \& Liukkonen, J. (2016). Fundamental movement skills and physical fitness 
as predictors of physical activity: A 6 year follow up study. Scandinavian Journal of Medicine \& Science in Sports, 26(1), 74-81

Jekauc, D., Wagner, M., Herrmann, C., Hegazy, K., \&Woll,A. (2017). Does physical self-concept mediate the relationship between motor abilities and physical activity in adolescents and young adults?. PLOS one, 12(1), e0168539.

Larsen, L., Kristensen, P., Junge,T., Rexen, C. , \&Wedderkopp, N. (2015). Motor performance as predictor of physical activity in children:The CHAMPS study-DK. Medicine and science in sports and exercise, 47(9), 1849-1856.

Lima, R., Pfeiffer, K., Larsen, L., Bugge, A., Moller, N., Anderson, L., \& Stodden, D. (2017). Physical activity and motor competence present a positive reciprocal longitudinal relationship across childhood and early adolescence. Journal of Physical activity and Health, 14(6), 440-447.

Lopes, L., Silva Mota, J., Moreira, C., Abreu, S., Agostinis Sobrinho, C., Oliveira-Santos, J., ... \& Santos, R. (2019). Longitudinal associations between motor competence and different physical activity intensities: LabMed physical activity study. Journal of sports sciences, 37(3), 285-290

Lubans, D., Morgan, P., Cliff, D., Barnett, L., Okely,A. (2010). Fundamental movement skills in children and adolescents. Sports Med. doi: 10.2165/11536850-00000000000000. PMID: 21058749.

Margozzini, P., \& Passi, Á. (2018). Encuesta Nacional de Salud, ENS 2016-2017: un aporte a la planificación sanitaria y políticas públicas en Chile. ARS MEDICA Revista de Ciencias Médicas, 43(1), 30-34.

Marín, P., \& López, N. (2020). Asociación de la competencia en las habilidades motrices básicas con las actividades físico-deportivas extracurriculares y el índice de masa corporal en preescolares. / Association of the fundamental movement skills competence with the extracurricular sports and the body mass index in preschoolers. Retos: nuevas tendencias en educación física, deporte y recreación, (38), 9.

Martin, E., True, L., Pfeiffer, K., Siegel, S., Branta, C., Wisner, D., ... \& Seefeldt, V. (2021). An examination of sport participation tracking and adult physical activity for participants of the Michigan State University motor performance study. Measurement in Physical Education and Exercise Science, 25(1), 35-42.

O’Brien,W., Belton, S., \& Issartel, J. (2016). Fundamental movement skill proficiency amongst adolescent youth. Physical Education and Sport Pedagogy, 21(6), 557 571

OMS (2020). Obesidad y sobrepeso. Consultado el 24 mayo 2020 en : https: / / www.who.int/es/news-room/factsheets/detail/obesity-and-overweight
Oñate, C., Batalla Flores,A., \& Páez, J. (2020). Elaboración y validez de un cuestionario de las habilidades motrices iniciales para estudiantes de enseñanza media chilena. Retos. Nuevas tendencias en Educación Física, Deporte y Recreación, 2020, vol.38, p. 465-471.

Pill, S., \& Harvey, S. (2019). A narrative review of children's movement competence research 1997-2017. Physical Culture and Sport. Studies and Research, 81(1), 47-74.

Rainer, P., \& Jarvis, S. (2020). Fundamental movement skills and their relationship with measures of health-related physical fitness of primary school children prior to secondary school transition: A Welsh perspective. Education 3-13, 48(1), 54-65.

Rodrigues, L., Luz, C., Cordovil, R., Bezerra, P., Silva, B., Camões, M., \& Lima, R. (2019). Normative values of the motor competence assessment (MCA) from 3 to 23 years of age. Journal of science and medicine in sport, 22(9), 10381043

Smith, L., Fisher, A., \& Hamer, M. (2015). Prospective association between objective measures of childhood motor coordination and sedentary behaviour in adolescence and adulthood. International Journal of behavioral nutrition and physical activity, 12(1), 1-6.

Timler, A., McIntyre, F., Bulsara, C., Rose, E., \& Hands, B. (2020). The influence of motor competence on adolescent identity health: A mixed method study. Research quarterly for exercise and sport, 91(1), 1-14.

Utesch, T., Bardid, F., Büsch, D., \& Strauss, B. (2019). The relationship between motor competence and physical fitness from early childhood to early adulthood: A metaanalysis. Sports Medicine, 49(4), 541-551.

Vallence, A., Hebert, J., Jespersen, E., Klakk, H., Rexen, C., \&Wedderkopp, N. (2019). Childhood motor performance is increased by participation in organized sport: the CHAMPS Study-DK. Scientific reports, 9(1), 1-8.

Van Niekerk, L. L., DuToit, D., \& Pienaar,A. E. (2016). The correlation between motor proficiency and physical activity in Senior Phase learners in the Potchefstroom area. health sa gesondheid, 21, 348-355

Werneck,A., Fernandes, R., \& Silva,D. (2020). Understanding biological maturation and motor competence for physical activity promotion during the first years of life. Translational pediatrics, 9(1), 1.

Zueck, M., García,A.,Villalobos, J., \& Gutiérrez, H. (2020). Satisfacción en las clases de Educación Física y la intencionalidad de ser activo en niños del nivel de primaria. Retos: nuevas tendencias en educación física, deporte y recreación, (37), 33-44 